\title{
Application of Edman's Phenylisothiocyanate Method in the Determination of C-terminal Amino Acids and Peptides after Hydrazinolysis *
}

\author{
PER WALLEN and INGVAR SJÖHOLM**
}

Chemistry Department II, Karolinska Institutet, Stockholm, Sweden

\begin{abstract}
Edman's phenylisothiocyanate method has been used for identification and quantitative determination of the C-terminal amino acids released in hydrazinolysis of fibrinopeptides $A$ and $B$ and of insulin. The method is found to be particularly suitable in cases where relatively large amounts of C-terminal peptides are formed, since it can also be used for sequence determinations of these peptides. For more detailed studies of complicated hydrazinolysates, Edman's method can be preceded by electrophoretic separation. The C-terminal sequences of fibrinopeptides $A$ and $B$ and of insulin as found in the present work are in accordance with the known structure of these substances.

Certain peptide bonds are found to be highly resistant to hydrazine under the conditions used $\left(100^{\circ} \mathrm{C}\right.$ for $\left.10 \mathrm{~h}\right)$. In hydrazinolysis of fibrinopeptide A, only an inappreciable quantity ( $5 \%$ ) of ornithine is obtained from the C-terminal amino acid arginine, whereas a good yield (about $70 \%$ ) is obtained from the di-and tripeptides val-orn and gly-val-orn, respectively, deriving from the C-terminal end. Thus, the peptide bond val-orn (val-arg) seems to be only inappreciably split in hydrazinolysis. The C-terminal peptide bond in fibrinopeptide B, ala-orn (ala-arg), also seems to be relatively resistant, as is the penultimate peptide bond in the B-chain of insulin, pro-lys.

The phenylthiohydantoin (P'H) derivative of ornithine has been synthesized. A solvent system is described for separation of the phenylthiohydantoin derivatives of lysine and ornithine.
\end{abstract}

A fter the introduction by Akabori et al..$^{1}$ of the hydrazinolysis method for determination of C-terminal amino acids in peptides and proteins, this technique has been applied in many investigations. The method elaborated by $\mathrm{Ohno}^{2}$ for identification and determination of the amino acids obtained after hydrazinolysis as the dinitrophenyl derivatives has subsequently been

* This work has been supported by Research Grant No. H-4295 (R 1) to Professor E. Jorpes from the National Heart Institute, National Institutes of Health, United States Public Health Service, and by grants from Karolinska Institutets Reservationsanslag, Svenska Sällskapet för Medicinsk Forskning and Svenska Nationalföreningen mot tuberkulos och andra folksjukdomar.

** Present address: Pharmacognosy Department, Kungl. Farmaceutiska Institutet, Stock. holm, Sweden.

Acta Chem. Scand. 14 (1960) No. 8 
adopted by many workers, particularly in the modification of Niu and FraenkelConrat $^{3}$. In view of the finding of Ohno ${ }^{2,4}$ and of Niu and Fraenkel-Conrat ${ }^{3}$ that C-terminal peptides are formed both on partial hydrazinolysis and when the C-terminally situated peptide bonds are resistant to hydrazine, the question arose of whether the phenylisothiocyanate method of Edman ${ }^{5}$ could be applied in the analysis of amino acids and peptides obtained on hydrazinolysis. In the determination of the amino acid sequence of peptides, this method is more advantageous than the dinitrophenyl (DNP) method most often used hitherto.

An account is given in the present paper of the application of Edman's method in C-terminal analyses of fibrinopeptides $A$ and $B$ and of insulin by the hydrazinolysis method, as well as of some observations on the resistance of certain peptide bonds to hydrazine. The phenylisothiocyanate method as modified by Sjoquist ${ }^{6-8,22}$ was used.

\section{EXPERIMENTAL}

\section{Materials}

Fibrinopeptide $A^{*}$.

Fibrinopeptide $B^{*}$.

Insulin, recrystallized 7 times**.

Hydrazine, anhydrous. Prepared according to Kusama ${ }^{10 * * *}$. $50 \mathrm{~g}$ of $99 \% \mathrm{~N}_{2} \mathrm{H}_{4} \cdot \mathrm{H}_{2} \mathrm{O}$, $500 \mathrm{~g}$ of crushed $\mathrm{CaO}$ and $500 \mathrm{~g}$ of toluene were allowed to stand overnight. The mixture was then refluxed for $3 \mathrm{~h}$ and distilled. An azeotropic mixture was obtained, consisting of toluene and hydrazine, b.p. $89-92^{\circ} \mathrm{C}$. The hydrazine content was determined with $\mathrm{KIO}_{3}$, using the method of Kolthoff ${ }^{11}$. Content: 98.2-99.5\%. Fresh hydrazine was prepared for each hydrazinolysis.

n-Heptaldehyde, technical, Hopkin \& Williams. Distilled in vacuo.

n-Heptane, Eastman-Kodak. Distilled, b.p. $98^{\circ} \mathrm{C}$.

5-[3-( $\beta$-Phenylthioureido)-propyl]-3-phenyl-2-thiohydantoin (Ornithine-PTH).

Prepared according to Edman's method for preparation of lysine-PTH ${ }^{12}$. The extinction curve showed a maximum at $\lambda=268 \mathrm{~m} \mu(\varepsilon=27650)$. $E^{245} / E^{260}=0.71$. M.p. $196-7^{\circ} \mathrm{C}$ (decomp.). (Found: N 14.45\%. Calc. 14.57).

n-Pentane, A. R., Kebo, Stockholm.

1,2-Dichloroethane, Hopkin \& Williams. Distilled, b.p. $83-84^{\circ} \mathrm{C}$.

Ethyl acetate, Hopkin \& Williams. Distilled, b.p. $77^{\circ} \mathrm{C}$.

Formic acid, A. R., Merck.

\section{Methods}

Hydrazinolysis. Before hydrazinolysis, the peptide material was dried in vacuo for $8-10 \mathrm{~h}$ in a desiccator over phosphorus pentoxide. $100 \mu \mathrm{l}$ of $\mathrm{N}_{2} \mathrm{H}_{4}$ were used for $3 \mathrm{mg}$ of peptide, $200 \mu \mathrm{l}$ for $12 \mathrm{mg}$, and $500 \mu \mathrm{l}$ for $55 \mathrm{mg}$. Four-ml test tubes with groundglass stoppers were used for hydrazinolysis, which was carried out for $10 \mathrm{~h}$ at $100^{\circ} \mathrm{C}$. The excess $\mathrm{N}_{3} \mathrm{H}_{4}$ was evaporated in vacuo over concentrated sulphuric acid for $2-3 \mathrm{~h}$. After this time, no smell of hydrazine was present.

Aldehyde treatment. The hydrazides formed were removed by shaking with $n$-heptaldehyde, as recommended by Kusama ${ }^{10}$. The preparations were dissolved in $0.5 \mathrm{ml}$

* Prepared according to Ref.' and kindly placed at our disposal by Dr. B. Blombäck, Karolinska Institutet, Stockholm.

** Kindly placed at our disposal by Mr. G. Lindén, Apoteksvarucentralen Vitrum, Stockholm. *** Hydrazine prepared according to Stähler ${ }^{13}$ or Locker ${ }^{14}$ had no advantages, both the relative quantity of C-terminal amino acids obtained and the total yield were smaller. 
of water, shaken three times with $0.75 \mathrm{ml}$ of $n$-heptane $+n$-heptaldehyde (1 : 1) for 5 ; 30 and $30 \mathrm{~min}$, after which the aqueous phase was freed from heptaldehyde by shaking twice with $0.5 \mathrm{ml}$ of heptane. Finally, it was shaken twice with $0.5 \mathrm{ml}$ of $n$-pentane. The aqueous phase was evaporated in vacuo over $\mathrm{P}_{2} \mathrm{O}_{5}$. The dried preparations were kept at $-15^{\circ} \mathrm{C}$.

The addition of heptane during the aldehyde treatment results in a larger yield of amino acids and peptides in the aqueous phase. In order to minimize the risk of the hydrazides being hydrolyzed, the first treatment with heptane-heptaldehyde should be carried out quickly.

Sequence determination. The amino acid sequence in the peptides was determined with the phenylisothiocyanate method of Edman, as modified by Sjöquist ${ }^{\mathbf{6}-8,22}$. The peptide material was treated with phenylisothiocyanate in a buffer solution of $\mathrm{pH}$ 10 for $1 \mathrm{~h}$ at $40^{\circ} \mathrm{C}$, resulting in formation of the phenylthiocarbamyl (PTC) derivatives of the peptides. The samples were washed with heptane and dried in vacuo $(<0.01 \mathrm{~mm}$ $\mathrm{Hg}$ ). The phenylisothiocyanate treatment was repeated once. The phenylthiohydantoin (PTH) derivatives of the N-terminal amino acids were obtained from the PTC peptides by treating them with a mixture of acetic acid saturated with hydrogen chloride and water $(2: 1)$ at $40^{\circ} \mathrm{C}$ for $21 / 2 \mathrm{~h}$. The samples were then re-dried in vacuo.

A mixture of dichloroethane, ethyl acetate and $n$-heptane $(2: 2: 1.5)$ with a density slightly lower than that of water, was washed 3 times with an equal volume of water. The last washing water (aqueous phase) was used to dissolve the vacuum-dried samples, after which the PTH derivatives were extracted twice with $0.75 \mathrm{ml}$ and three times with $0.5 \mathrm{ml}$ of the solvent mixture. The organic phase was evaporated by a stream of air, and the aqueous phase in vacuo over $\mathrm{P}_{\mathbf{2}} \mathrm{O}_{\mathrm{B}}$.

The dried aqueous phase, which contained the remaining part of the peptide, could then be re-treated with phenylisothiocyanate, after which the procedure already described was repeated. All operations, from hydrazinolysis to extraction of the PTH derivatives inclusively, were performed in the same test tube.

The PTH derivatives were identified and determined quantitatively by paper chromatography, according to Sjöquist ${ }^{8}$. On hydrazinolysis arginine is converted to ornithine. In Sjöquist's system, ornithine-PTH migrates with lysine-PTH and glycine-PTH in solvent II, and with lysine-PTH and alanine-PTH in solvent III. Consequently, ornithine cannot be determined separately from lysine. In order to permit concurrent determination of the two basic amino acids separately in a mixture, we used a solvent system consisting of $n$-heptane, dichloroethane and $75 \%$ formic acid $(1: 1: 2)$. The organic phase was used as eluent, and was also sprayed over the strips of filter paper on the short ends of a chromatography tank of the kind described by Sjöquist ${ }^{8}$. The formic acid phase (stationary phase) was sprayed over the strips of filter paper hanging in the tank. Each time the tank was used, the old stationary phase was replaced by a fresh one. One hour was required for equilibration. With this system, the PTH-derivatives of glycine, ornithine, lysine and alanine could be completely separated (see Fig. 1).

Paper electrophoresis. Electrophoresis of the peptide material obtained after hydrazinolysis and heptaldehyde treatment was performed with a water-cooled high-voltage apparatus of Perspex. A buffer consisting of $6 \mathrm{ml}$ of glacial acetic acid and $8 \mathrm{ml}$ of pyridine in 1 liter of water ( $\mathrm{pH} 4.9$ ) was used throughout. Whatman No. 3 MM paper was used for preparative electrophoresis. A potential of $25 \mathrm{~V} / \mathrm{cm}$ was applied across the paper, resulting in a current intensity of approximately $30 \mathrm{~mA}$. Adequate separation was obtained after about $11 / 2 \mathrm{~h}$.

The preparation was placed on a $5 \mathrm{~cm}$ wide strip. In order to identify the fractions, a small quantity of the preparation was applied as a spot beside the main part of it. After electrophoresis and drying at $100^{\circ} \mathrm{C}$, a strip corresponding to this spot was cut out, and developed with a ninhydrin reagent $(0.25 \%$ ninhydrin $+0.5 \%$ collidine in $95 \%$ ethanol). The fluorescence of the substances in ultraviolet light was also used for identification purposes. The fractions were eluted with water, and the eluates evaporated in vacuo over $\mathrm{P}_{2} \mathrm{O}_{5}$.

Hydrolysis. Hydrolysis of the peptides was carried out in constantly boiling hydrochloric acid at $105^{\circ} \mathrm{C}$ for $23 \mathrm{~h}$, using evacuated sealed glass tubes.

Two-dimensional paper chromatography. Two-dimensional paper chromatography, performed on both unhydrolyzed and hydrolyzed electrophoretic fractions, was done on Whatman No. 1 paper. Development of the chromatograms took place in the first direc-

Acta Chem. Scand. 14 (1960) No. 8 


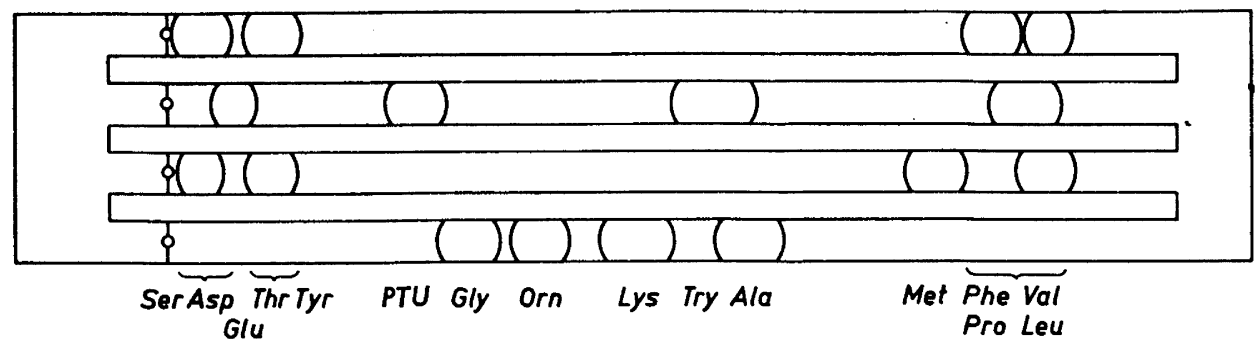

Fig. 1. Separation of PTH derivatives of glycine, ornithine, lysine and alanine. Solvent: $n$-heptane-dichloroethane $-75 \%$ formic acid $(1: 1: 2)$.

tion (descending) with the upper phase of a mixture of acetic acid, $n$-butanol and water $(1: 4: 5)$, and in the second direction (ascending) with $80 \%$ pyridine in water.

Quantitative amino acid analysis. Quantitative analysis of the amino acids in hydrolyzed material was performed according to Sjöquist •

\section{RESULTS}

Fibrinopeptide $A$. Hydrazinolysis according to the standard procedure described in the aforegoing was performed on about $1 \mu$ mole $(1.89 \mathrm{mg}$ ) of fibrinopeptide A. After removal of the hydrazides, sequence determination was made on the C-terminal fraction, without previous separation by electrophoresis or paper chromatography. The results are shown in Table 1 . In the first step, glycine and valine were obtained as the main components. On the other hand, ornithine - which on hydrazinolysis is formed from arginine appeared only in extremely small quantities, and was completely lacking in some experiments. This is remarkable, since it has recently been demonstrated that the C-terminal sequence in fibrinopeptide $\mathrm{A}$ is gly-val-arg ${ }^{15,16}$. In the second and third steps, on the contrary, ornithine appeared in considerably larger quantities, amounting to $70-80 \%$ of the valine in the preceding step. The valine in the second step corresponded fairly well to the glycine in the first step. In the fourth step, no PTH amino acids could be demonstrated. These experiments seemed to afford definite evidence that hydrazinolysis results in formation chiefly of the C-terminal residues gly-val-orn and val-orn, whereas only extremely small quantities of the C-terminal amino acid are present.

Table 1. Amino acid sequence determination of the heptaldehyde-treated hydrazinolysate of fibrinopeptide A. The values are not corrected for the loss in hydrazinolysis and heptaldehyde treatment.

\begin{tabular}{|c|c|c|c|c|}
\hline Amino & \multicolumn{4}{|c|}{ Mole PTH derivative per mole fibrinopeptide A } \\
\hline acid & Step 1 & Step 2 & Step 3 & Step 4 \\
\hline gly & 0.35 & - & - & Trace \\
\hline val & 0.34 & 0.22 & - & - \\
\hline orn & 0.05 & 0.27 & 0.19 & - \\
\hline pro & 0.08 & - & - & - \\
\hline asp & 0.03 & 0.04 & - & - \\
\hline glu & 0.05 & - & - & Trace \\
\hline ser & 0.03 & Trace & - & - \\
\hline
\end{tabular}




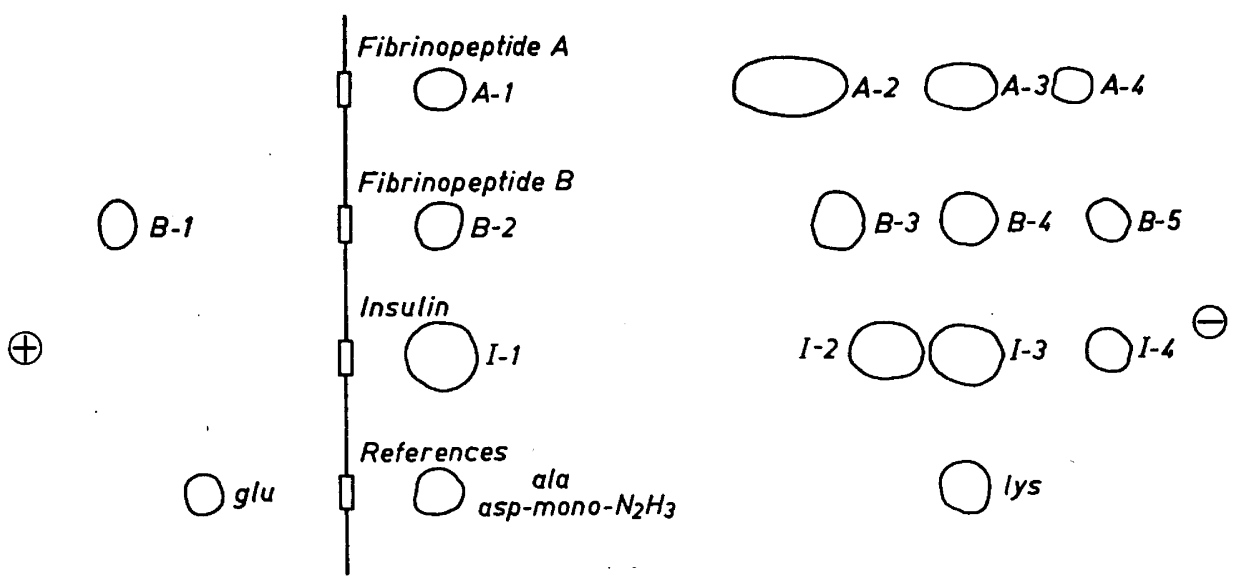

Fig. 2. Electropherograms of hydrazinolyzed and heptaldehyde-treated fibrinopeptides $\mathrm{A}$ and $\mathrm{B}$ and insulin $(\mathrm{pH}=4.9, \mu=0.1$ and length of runs $90 \mathrm{~min}$ at $25 \mathrm{~V} / \mathrm{cm}$ in all figures).

For additional verification of these results, attempts were made to separate the components of the hydrazinolysate freed from the hydrazides, by means of electrophoresis and paper chromatography. About $8 \mu$ moles $(15 \mathrm{mg})$ of fibrinopeptide A were hydrazinolyzed with $0.2 \mathrm{ml}$ of hydrazine. The excess hydrazine and the hydrazides were removed as described earlier. In electrophoresis, four fractions could be identified (Fig. 2); they all migrated to the cathode. In the following, they are denoted as A-1, A-2, A-3, and A-4.

A-1, which migrated at the same rate as the neutral amino acids, and A-2, which migrated somewhat more slowly than lysine, were consistently present. A-3, at the site of lysine and ornithine, and A-4, which was strongly basic and could be identified only by its UV fluorescence, were weak and were sometimes lacking altogether.

The fractions were eluted from the paper, divided into two equal parts, and dried. One part of each electrophoretic fraction was used for two-dimensional paper chromatography and for sequence determination (Fig. 3 and Table 2). The other part was hydrolyzed, after which the component amino acids were identified by means of two-dimensional paper chromatography.

In two-dimensional paper chromatography, fraction A-1 showed only two weak components, one of which was probably glycine (Fig. 3). After hydrolysis, on the other hand, several amino acids appeared, i.e. proline, as well as traces of serine, aspartic acid, glutamic acid and glycine. Sequence analysis of the unhydrolyzed part of the fraction disclosed that all these amino acids already appeared in the first step, and serine and aspartic acid in the second step as well (Table 2).

Fraction A-2 was shown by two-dimensional paper chromatography to consist of two strong components, which were partly confluent (Fig. 3). Owing to lack of material, no attempt was made to separate and analyze these components individually. After hydrolysis of this fraction, large quan-

Acta Chem. Scand. 14 (1960) No. 8 

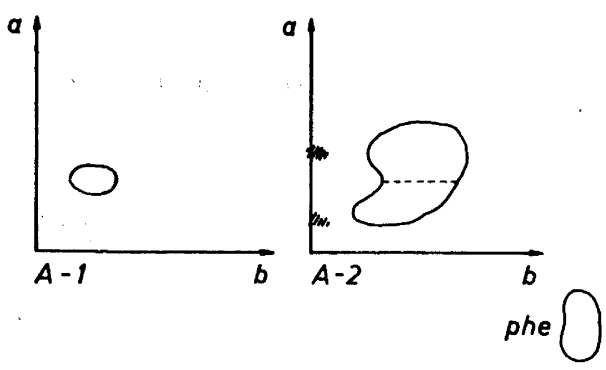

val

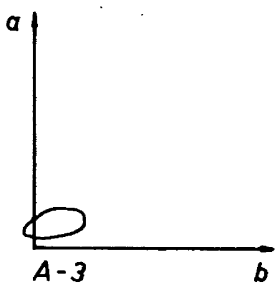

Fig. 3. Two-dimensional paper chromatograms of the electrophoretic fractions A-1, $A-2$, and A-3, obtained from hydrazinolyzed and heptaldehyde-treated fibrinopeptide $\mathbf{A}$. Solvent a: $n$-butanol-acetic acid-water (4 : $1: 5)$. Solvent b: pyridine-water $(4: 1)$. Traces are indicated by shaded areas.

tities of glycine, valine and ornithine could be demonstrated. In sequence determination, only glycine and valine appeared in the first step (Table 2). In the second step, valine and ornithine appeared in quantities corresponding approximately to the glycine and valine, respectively, in the first step. No glycine appeared in this step. In the third step, ornithine appeared in quantities corresponding to the valine in the second step. No other amino acids could be identified in this step. Fraction A-2 thus seemed to consist of two peptides, val-orn and gly-val-orn.

Table 2. Amino acid sequence determination of electrophoretic fractions from heptaldehyde-treated hydrazinolysate of fibrinopeptide $A$. The figures denote the number of $\mu$ moles obtained from about $4 \mu \mathrm{moles}(7.6 \mathrm{mg})$ of fibrinopeptide A. The values are not corrected for the loss in hydrazinolysis, heptaldehyde treatment and electrophoresis.

Fraction Amino acid Step $1 \quad$ Step $2 \quad$ Step 3

\begin{tabular}{llcccc}
\hline A-1 & pro & 0.09 & - & - \\
& ser & 0.03 & 0.03 & - \\
& asp & 0.03 & 0.03 & - \\
& glu & 0.06 & - & - \\
& gly & Trace & - & - \\
& gly & 0.60 & $\overline{0}$ & - \\
& A-2 & val & 0.92 & 0.41 & $\overline{-}$ \\
& orn & - & 0.92 & 0.47 \\
\hline & orn & 0.08 & - & - \\
\hline \multirow{2}{*}{$\mathrm{A}-3$} & - & & & \\
\hline
\end{tabular}


Table 3. Amino acid sequence determination of the heptaldehyde-treated hydrazinolysate of fibrinopeptide B. The values are not corrected for the loss in hydrazinolysis and heptaldehyde treatment.

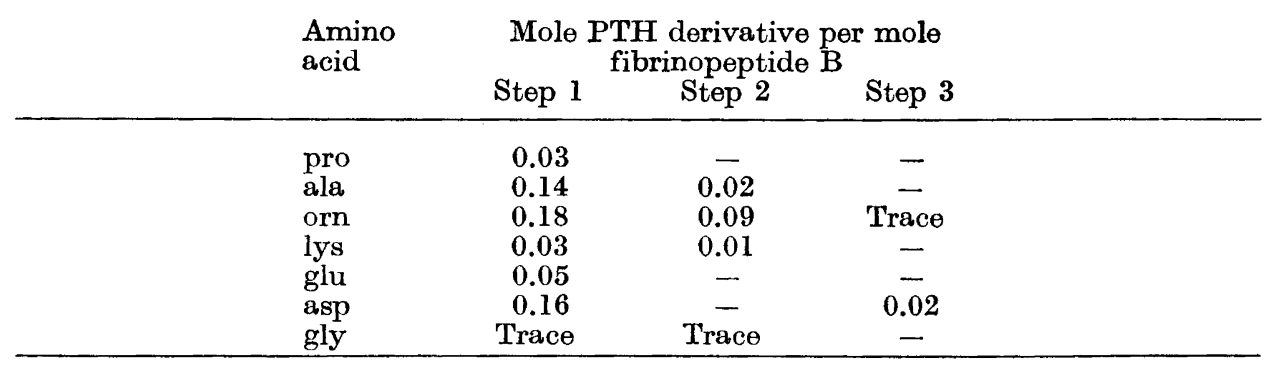

In paper chromatography of fraction A-3, only one spot at the site of lysine and ornithine was obtained before hydrolysis (Fig. 3). The result was the same after hydrolysis. Sequence determination gave ornithine alone in the first step, and no amino acids in the two subsequent steps. This fraction thus consisted only of ornithine. Quantitatively, however, it amounted to only a small proportion (6-7\%) of the ornithine in the C-terminal fragments of A-2.

Fraction A-4 gave no ninhydrin-positive spot in direct paper chromatography. After hydrolysis, one weak spot at the site of lysine and ornithine was obtained in two-dimensional chromatography. No PTH derivatives of these amino acids were, however, obtained in determination with Sjöquist's method.

Fibrinopeptide $B$. Fibrinopeptide $\mathrm{B}$ was studied in largely the same way as fibrinopeptide A. Sequence determinations were made on the hydrazinolysate remaining after heptaldehyde treatment. The results of one such test are shown in Table 3.1 In the first step, ornithine, was obtained which agrees with arginine being the C-terminal amino acid. In addition, alanine and aspartic acid appeared in quantities that could not be neglected. The presence of alanine might indicate that the C-terminal dipeptide was present in the sample. As has been shown recently, the C-terminal sequence in fibrinopeptide $\mathrm{B}$ is gly-ala-arg ${ }^{15,16}$. In the second step, it was chiefly ornithine that appeared. This may agree with the sequence ala-arg. However, in view of the high aspartic acid value in the first step, the ornithine might equally well have derived from an asp-arg sequence.

In order to obtain further information, paper electrophoresis was performed on the heptaldehyde-treated hydrazinolysate. The appearance of the electropherograms is shown in Fig. 2. Five fractions were identified; they are denoted in the following as B-1, B-2, B-3, B-4, and B-5. B-1 had a negative charge, and all the other fractions a positive charge.

The fractions were eluted with water. Two-dimensional paper chromatography was performed on a small part of each fraction (Fig. 4). Except for traces of glycine in fraction B-2, and spots corresponding to ornithine or lysine in B-3 and B-4, no spots indicative of free amino acids were present. The spots in fraction B-5 could not have been free amino acids, since in electrophoresis

Acta Chem. Scand. 14 (1960) No. 8 

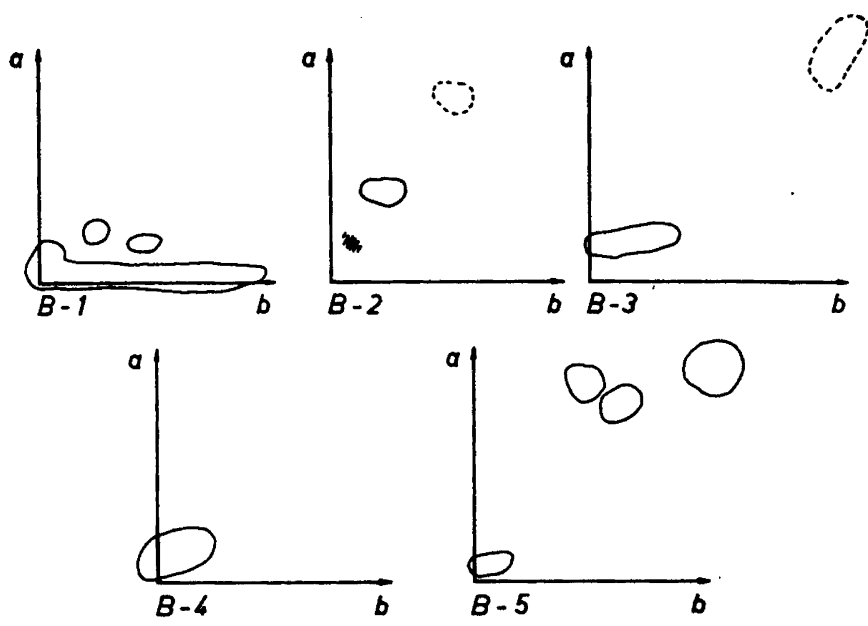

Fig. 4. Two-dimensional paper chromatograms of the electrophoretic fractions B-1, B-2, B-3, B-4, and B-5, obtained from hydrazinolyzed and heptaldehyde-treated fibrinopeptide B. (The solvent system is the same as in Fig. 3.) Faint spots are indicated by dotted lines, traces by shaded areas.

this fraction migrated further than the amino acids conceivably present in the sample.

Part of each electrophoretic fraction was removed and hydrolyzed. Quantitative amino acid analysis according to Sjöquist was then done on the hydrolysate. The results are listed in Table 4. In fractions B-1 and B-2, glutamic acid and aspartic acid predominated. Traces of ornithine and glycine were also demonstrable. Fraction B-3 was found to consist mainly of equally large quantities of alanine and ornithine, in addition to extremely small quantities of lysine, glutamic acid and aspartic acid.

In sequence analysis of the unhydrolyzed fraction B-3, alanine alone appeared in the first step, and ornithine in the second step. It is evident that this fraction consisted chiefly of the dipeptide ala-orn, which must have derived from the C-terminal dipeptide ala-arg. Both before and after hydrolysis, frac-

Table 4. Quantitative determination of the amino acid content of the electrophoretic fractions of hydrazinolyzed and heptaldehyde-treated fibrinopeptide B. The figures denote the number of $\mu$ moles obtained from about $3.3 \mu$ moles $(8.2 \mathrm{mg})$ of fibrinopeptide B. The values are not corrected for the loss in hydrazinolysis, heptaldehyde treatment and electrophoresis.

\begin{tabular}{lccccc}
$\begin{array}{l}\text { Amino } \\
\text { acid }\end{array}$ & B-1 & B-2 & $\begin{array}{c}\text { Fraction } \\
\text { B -3 }\end{array}$ & B-4 & B-5 \\
\hline ala & - & $-\overline{07}$ & 0.40 & Trace & $-\overline{14}$ \\
orn & 0.07 & 0.06 & 0.43 & 0.53 & 0.14 \\
lys & $\overline{-}$ & $\overline{-}$ & 0.07 & 0.08 & - \\
gly & 0.13 & 0.29 & $\overline{-}$ & $\overline{-}$ & 0.04 \\
glu & 0.24 & 0.47 & 0.04 & 0.04 & Trace \\
asp & 0.36 & 0.87 & 0.03 & Trace & \\
\hline
\end{tabular}


tion B-4 yielded only large quantities of ornithine, in addition to traces of alanine and lysine. This fraction thus consisted mainly of the ornithine derived from the C-terminal amino acid arginine. After hydrolysis of fraction B-5, ornithine appeared, probably derived from the ornithine hydrazide.

In some experiments with fibrinopeptide B, PTH proline appeared in fairly large quantities in the first step of sequence determination of the unfractionated hydrazinolysate. In these cases, approximately equimolar amounts of the PTH derivatives of lysine and valine were obtained in the second and third step, respectively. In the fourth step, glycine could also be traced, but was not determined quantitatively. In qualitative amino acid determinations of the electrophoretic fractions after hydrolysis, fraction B-4 was found to contain proline, lysine, valine and glycine, in addition to the ornithine normally present. Fraction B-3 also contained these amino acids, although in smaller amounts, which made the quantitative determinations unreliable.

In determination of the sequences in these two fractions, proline, lysine, valine, and glycine appeared in the first, second, third, and fourth step, respectively. It is possible that, in these experiments, a peptide hydrazide was formed with the sequence pro-lys-val-gly, which was present in the electrophoretic fraction B-4. In this event, the hydrazide would have been partly hydrolyzed during the procedure, resulting in formation of the corresponding peptide, present in fraction B-3. The reason why this hydrazide was not removed by aldehyde treatment is uncertain. A possible explanation is that, because of its size and charge, this hydrazide had become so hydrophilic that it remained in the aqueous phase on treatment with aldehyde. We did, in fact, also obtain small quantities of ornithine and lysine hydrazides in the electrophoretic fractions B-5 and I-4 (see the following), which indicates that the basic hydrazides are not, as a rule, completely removed by aldehyde treatment.

Insulin. Sequence determination with the procedure described earlier was performed on the heptaldehyde-treated hydrazinolysate from about $2 \mu$ moles $(11.5 \mathrm{mg}$ ) of insulin. The results are summarized in Table 5. Each value is the mean of the values obtained in 14 hydrazinolyses with only small differences between them. As could be expected, alanine appeared in relatively large quantities in step 1 , but measurable amounts of proline, lysine and ornithine could also be identified, as well as traces of glycine, glutamic acid and aspartic acid. Since the B-chain of insulin ends with pro-lys-ala ${ }^{17}$, it seemed likely that the lysine and proline demonstrable in step 1 comprised the N-terminals of the C-terminal peptides formed in hydrazinolysis. The subsequent analysis did not verify this assumption, since the amount of alanine in steps 2 and 3 by no means corresponded to the quantity of lysine obtained in the preceding step. The results of the sequence determination indicated instead that the hydrazinolysate contained - in addition to small quantities of C-terminal peptides, lys-ala and pro-lys-ala - free lysine and possibly some proline, as well as the peptide pro-lys.

For further elucidation, electrophoretic separation of the beptaldehydetreated hydrazinolysate was performed, as well as subsequent analysis of the various fractions. In electrophoresis, we obtained four fractions - in one case a fifth as well (Fig. 2); they are denoted in the following as I-1, I-2, I-3, I-4, and I-5. All fractions migrated to the cathode. I-5, which was not invariably

Acta Chem. Scand. 14, (1960) No. 8 
Table 5. Amino acid sequence determination of the heptaldehyde-treated hydrazinolysate of insulin. The values are not corrected for the loss in hydrazinolysis and heptaldehyde treatment.

\begin{tabular}{lcccc} 
& Amino & \multicolumn{4}{c}{ Mole PTH derivative per mole insulin } \\
acid & Step 1 & Step 2 & Step 3 \\
\hline ala & 0.38 & Trace & Trace \\
lys & 0.07 & 0.10 & - \\
pro & 0.22 & Trace & - \\
orn & 0.04 & Trace & - \\
gly & Trace & - & $=$ \\
glu & Trace & - & - \\
asp & Trace & - & - \\
\hline
\end{tabular}

present, moved further than lysine, and probably consisted of strongly basic hydrazides, which had not been completely removed by aldehyde treatment. No analysis was therefore made of this fraction.

The various electrophoretic fractions were chromatographed both before (Fig. 5) and after hydrolysis in the two-dimensional chromatography system described.

In electrophoresis, fraction I-1 migrated as far as the neutral amino acids and the glutamic and aspartic acid monohydrazides. Chromatographically, it consisted of two fractions. One of them migrated at the site of alanine, and one at the site of glycine. No proline - which, if it had been present in free form, should have appeared in this fraction - was, however, observed. After hydrolysis, a fairly complicated pattern was obtained. In addition to alanine, which gave a strong spot, small quantities of aspartic acid, glycine, serine, lysine and/or ornithine were present, an well as traces of valine, proline and threonine. Thus, in I-1, alanine and possibly glycine migrated, whereas the other amino acids obtained on hydrolysis derived from small quantities of peptide hydrazides with one free carboxyl group, as well as aspartic and glutamic monohydrazides.
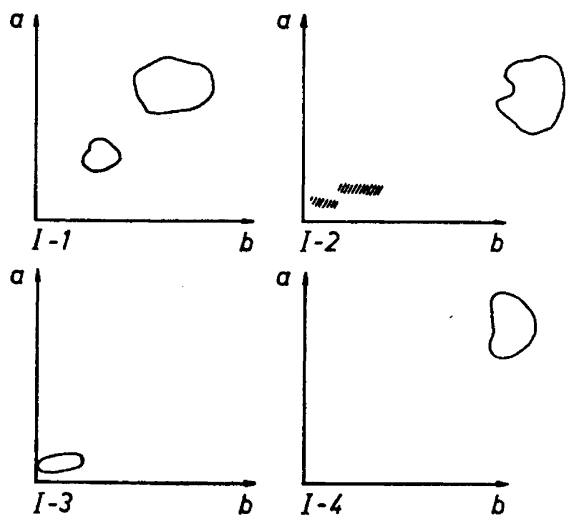

Fig. 5. Two-dimensional paper chromatograms of the electrophoretic fractions I-1, I-2, I-3, and I.4, obtained from hydrazinolyzed and heptaldehyde-treated insulin. (The solvent system is the same as in Fig. 3.) Traces are indicated by shaded areas. 
Electrophoretically, fraction I-2 migrated somewhat more slowly than lysine and ornithine. In chromatography of the fraction, we obtained a component which moved like alanine in the butanol direction, but somewhat more slowly than alanine in the pyridine direction. This component reacted strongly to ninhydrin. Two additional fractions, which reacted very weakly to ninhydrin, were present. They migrated close to the site of lysine. After hydrolysis, relatively large quantities of proline and lysine were obtained, as well as extremely small amounts of aspartic acid, glycine and alanine. This fraction thus seemed to consist chiefly of a peptide containing proline and lysine.

Fraction I-3 moved as rapidly in electrophoresis as lysine and ornithine. In our system, the fraction was found chromatographically uniform with a single spot at the site of lysine and ornithine. After hydrolysis, lysine and/or ornithine were demonstrable, as well as traces of aspartic acid and glycine. This fraction thus consisted of free lysine and/or ornithine.

In chromatography, fraction I-4 which yielded a yellow spot in the ninhydrin reaction, moved slightly more rapidly than alanine. After hydrolysis, it proved to consist of lysine and/or ornithine.

The analysis therefore showed that the bydrazinolysate consisted principally of alanine and pro-lys, as well as small amounts of lys-ala, lysine and ornithine. In addition to these peptides and amino acids, it contained small quantities of hydrazides of amino acids and peptides, which had not been removed completely through the aldehyde treatment. The presence of amino acids and peptides that were not C-terminal might imply that undesirable hydrolysis of hydrazides to free amino acids and peptides had occurred in the course of analysis. Previous investigations have, however, shown that if removal of the hydrazides is done rapidly, such hydrolysis takes place only to an inappreciable extent ${ }^{18}$. Another possible explanation is that in preparation of the insulin, it might have been partially split by the traces of trypsin present. Since the action of trypsin has been found to be strictly confined to peptide bonds in which the carboxyl groups of basic amino acids are included, such splitting could have taken place only in the B-chain, between the amino acids $22-23$ (arg-gly) and 29-30 (lys-ala) ${ }^{19}$. It is probable that the large fragments thus formed follow the unaltered insulin during the preparation. Those amino acids and peptides obtained in the analysis that can not have been released from the C-terminal sequence in the complete insulin molecule can all have derived from the C-terminal sequence in the fragments formed by the action of trypsin, i.e., glu-arg, which yields orn, and thr-pro-lys, which yields both pro-lys and lys. Evidently the recrystallized insulin used in this work was prepared with a technique in which a certain enzymatic destruction is unavoidable. That such an interaction of trypsin on insulin can occur in the process of manufacturing has since then been demonstrated by Jorpes and Lindén ${ }^{20}$. However heating of the crude alcoholic extract of the pancreatic gland at $\mathrm{pH} 2.0-4.5$ at a temperature of $70^{\circ}-75^{\circ} \mathrm{C}$ for 10 min will destroy the trypsin and considerably increase the yield in the large scale manufacture of insulin. The recrystallized insulin used in this work was in fact prepared with a technique in which a certain enzymatic destruction of insulin is unavoidable. 


\section{DISCUSSION}

When Edman's phenylisothiocyanate method is applied to a hydrazinolysate of a peptide, the C-terminal amino acid released is determined, as well as the $\mathrm{N}$-terminal amino acids of the C-terminal peptides formed in hydrazinolysis. By utilizing the possibility of sequence determination given by this method, the C-terminal sequence can, in favourable cases, be determined directly on the heptaldehyde-treated hydrazinolysate, without further separation. About $1-2 \mu$ moles of peptide material are required for such an analysis.

A direct sequence determination according to the aforegoing should give sufficiently reliable results in a relatively pure hydrazinolysate from peptides consisting of a single chain. In certain cases, however, it is not possible to obtain a clear picture of the C-terminal constitution of the peptide without further separation of the components of the hydrazinolysate. This applies, for example, in studies of peptides with more than one chain, and those whose structure disposes to the formation of acid peptide hydrazides, which are difficult to remove completely from the hydrazinolysate. By using high-voltage electrophoresis and two-dimensional paper chromatography, we were able to separate from the hydrazinolysate several fractions, which were subsequently analyzed.

Of the analyses performed in the present investigation, those on fibrinopeptide $\mathrm{A}$ and insulin gave results that are in agreement with the $\mathrm{C}$-terminal sequence of the peptides. In application of Edman's method to the hydrazinolysate of these peptides, we obtained exceedingly small - in certain cases not measurable - amounts of PTH derivatives, particularly from glycine, aspartic acid and glutamic acid, that could not have been released from the C-terminal parts. The quantities of these amino acids were about as large as those reported by Bradbury ${ }^{21}$ in his studies on insulin, i.e., as a rule $<0.05$ mole per mole of peptide. After electrophoresis and two-dimensional paper chromatography of the hydrazinolysate it could, in fact, be shown that with the possible exception of glycine - they did not derive from free amino acids. After hydrolysis of the less basic electrophoretic fractions, we nevertheless obtained small quantities of glutamic and aspartic acid. In view of the rate of migration of the electrophoretic fractions they should have derived from hydrazides. Glutamic and aspartic acid can occur inside a peptide chain with free carboxyl groups. In hydrazinolysis, the free carboxyl group remains intact, whereas a hydrazide group is obtained from the carboxyl group in the peptide bond. It is probable that, at the $\mathrm{pH}$ applied in heptaldehyde treatment, the free carboxyl group makes the hydrazone formed slightly hydrophilic, so that in certain cases it is not completely extracted with the organic heptane-heptaldehyde phase. In an attempt to produce more effective removal of the hydrazides, we substituted benzaldehyde for heptaldehyde in some experiments, but this did not prove to have any advantage.

The results obtained in hydrazinolysis of fibrinopeptide $B$ were less clear. The analyses indicated that the hydrazinolysate contained - in addition to ornithine and ala-orn, which may have derived from the C-terminal sequence - aspartic acid and glutamic acid hydrazides and, in some cases, also a tetrapeptide hydrazide with the sequence pro-lys-val-gly. The same sequence has been demonstrated in sequence studies of fibrinopeptide $\mathrm{B}^{22}$. 
Since some of the bydrazides formed in hydrazinolysis contain one or several free carboxyl groups, which make them extremely difficult to remove from the water phase, the value of this method for C-terminal analyses is presumably reduced in some cases. This is because certain difficulties may arise in studies of $e . g$. peptides and proteins containing large quantities of aspartic and glutamic acid. It is, however, possible that the use of solid polyaldehydes - e.g. polyacrolein as introduced by Kauffmann and Boettcher ${ }^{23}$ - would result in more effective removal of the hydrazides, and thus in a still more reliable method for C-terminal studies.

Our experiments were made on peptides containing basic amino acids in their C-terminal part. After 10 hours' treatment with hydrazine, we were able to demonstrate relatively large quantities of peptides. This might indicate that the peptide bonds in which basic amino acids are present are fairly resistant to hydrazine. Thus, we found, for example, in hydrazinolysis of fibrinopeptide $\mathrm{B}$ that the peptide ala-orn was obtained in almost as large a quantity as ornithine (Table 3). The lys-ala bond in the intact insulin molecule seems, on the contrary, to be fairly easily split, since only small amounts of lys-ala were demonstrable. On the other hand, large quantities of the dipeptide pro-lys were present, and small quantities of lys. This implies that the peptide bond pro-lys is also relatively resistant in hydrazinolysis. The resistance of the val-arg (orn) bond, which occurs C-terminally in peptide A, is still more marked. In analysis of fibrinopeptide $A$ we obtained, in fact, in the first step of sequence determination 0.36 mole of gly (from gly-val-orn) and 0.34 mole of val (from val-orn), but only 0.05 mole of orn per mole of peptide A. In this case, the amount of peptide is 14 times the amount of C-terminal amino acid. Our results seem to show that the gly-val bond is also relatively stable.

The number of hydrazinolysis studies is still too small to permit any definite conclusions regarding which peptide bonds are difficult to split, or the reason underlying the difference between the sensitivity of the bonds to hydrazine. No complete investigation has yet been made of the kinetics in hydrazinolysis of peptides, although Bradbury ${ }^{21}$ has studied the rate of hydrazinolysis in certain tri- and dipeptides as catalyzed by hydrazonium sulphate. He was able to demonstrate considerable differences between the stability of the peptides. For example, the peptide leu-val was much more stable than leu-gly which, in turn, showed greater stability than gly-gly. Judging by the material hitherto available for analysis, a peptide bond whose amino group derives from a basic amino acid is relatively stable, as are bonds containing amino acids with longer and branched carbon chains.

Our results indicate the existence of certain peptide bonds that are considerably more resistant to hydrazine than was formerly believed. If a peptide or a protein has resistant peptide bonds in its C-terminal part, the hydrazinolysate from them will contain a varying proportion of peptides even after relatively lengthy hydrazine treatment. If the C-terminal peptide bond is almost completely resistant - as, e.g. val-arg in fibrinopeptide A - the yield of the free C-terminal amino acid will be extremely small. Naturally, in such cases it is not possible to apply the criteria suggested by Akabori et al..$^{18}$ for evaluation of whether or not the amino acid is situated C-terminally. According to these criteria, the yield should be at least $20 \%$ of a certain amino acid

Acta Chem. Scand. 14 (1960) No. 8 
for it to be regarded as C-terminal. The value often falls below this figure, and the yield may even be so low that it is difficult to determine exactly. For example, Dixon, Kauffmann and Neurath ${ }^{24}$ recently described a peptide obtained by tryptic hydrolysis of oxidized di-isopropyl-phosphoryl-trypsin, and denoted as $\mathrm{O}-\mathrm{Tr}-1$, in which no $\mathrm{C}$-terminal amino acid could be determined by hydrazinolysis in the modification of Niu and Fraenkel-Conrat. They were, however, able to show by partial hydrolysis that the C-terminal sequence was gly-lys. Like ala-orn (arg) in fibrinopeptide $\mathrm{B}$, this peptide bond is probably relatively stable in hydrazinolysis.

Our knowledge of which amino acids or combinations of them determine the resistance of the peptide bonds to hydrazine is still incomplete. Consequently, a full analysis must be made of the hydrazinolysate obtained in order to get an accurate picture of the C-terminal structure of an unknown peptide. A suitable tool for such an analysis is Edman's phenylisothiocyanate method, possibly preceded by electrophoretic separation of peptides and amino acids in the aldehyde-treated hydrazinolysate. Edman's method seems to have certain advantages over other techniques hitherto available. This is because it gives a good quantitative picture of the composition of the hydrazinolysate, and also permits a stepwise breakdown of the existing peptides.

Acknowledgements. The authors' thanks are due to Professor Erik Jorpes. We feel much indebted to Docent John Sjöquist, Lund, for valuable advice and discussions.

\section{REFERENCES}

1. Akabori, S., Ohno, K. and Narita, K. Bull. Chem. Soc. Japan 25 (1952) 214.

2. Ohno, K. J. Biochem. 40 (1953) 621.

3. Niu, C. I. and Fraenkel-Conrat, H. J. Am. Chem. Soc. 77 (1955) 5882.

4. Ohno, K. J. Biochem. Tokyo 42 (1955) 615.

5. Edman, P. Acta Chem. Scand. 4 (1950) 283.

6. Sjöquist, J. Arkiv Kemi 11 (1957) 129.

7. Sjöquist, J. Arkiv Kemi 14 (1959) 291.

8. Sjöquist, J. Biochim. et Biophys. Acta. 41 (1960) 20.

9. Blombäck, B. and Vestermark, A. Arkiv Kemi 12 (1958) 173.

10. Kusama, K. J. Biochem. Tokyo 44 (1957) 375.

11. Kolthoff, I. M. J. Am. Chem. Soc. 46 (1924) 2009.

12. Edman, P. Acta Chem. Scand. 4 (1950) 277.

13. Stähler, A. Ber. 42 (1909) 3018.

14. Locker, R. H. Biochim. et Biophys. Acta 14 (1954) 533.

15. Blombäck, B., Sjöquist, J. and Wallén, P. Acta Chem. Scand. 13 (1959) 819.

16. Folk, J. E., Gladner, J. A. and Laki, K. J. Biol. Chem. 234 (1959) 67.

17. Sanger, F. and Tuppy, H. Biochem. J. 49 (1951) 481.

18. Akabori, S., Ohno, K., Ikenaka, T., Okada, Y., Hanafusa, H., Haruna, I., Tsugita, A., Sugoe, K. and Matsushima, T. Bull. Chem. Soc. Japan 29 (1956) 507.

19. Sanger, F. and Tuppy, H. Biochem. J. 49 (1951) 481.

20. Jorpes, J. E. and Lindén, J. G. B. Canadian patent 594 614, March 22, 1960.

21. Bradbury, J. H. Biochem. J. 68 (1958) 482.

22. Sjöquist, J., Blombäck, B. and Wallén, P. Arkiv Kemi. 16 (1960) 425.

23. Kauffmann, T. and Boettcher, F.-P. Ann. 625 (1959) 123.

24. Dixon, G. H., Kauffmann, D.-L. and Neurath, H. J. Biol. Chem. 233 (1958) 1373.

Received June 14, 1960. 